\title{
Fator de correção para indivíduos com capacidade acomodativa baseado no uso do refrator automático
}

\author{
Correction factor for individuals with accommodative capacity \\ based on automated refractor
}

\author{
Rodrigo Ueno Takahagi ${ }^{1}$ \\ Andréia Lavagnoli ${ }^{2}$ \\ Tatiana Moura Fernandes ${ }^{3}$ \\ Silvana Artioli Schelinni ${ }^{4}$ \\ Carlos RobertoPadovani ${ }^{5}$
}

\begin{tabular}{|l|}
\hline RESUMO \\
\hline Objetivo: Pesquisar um fator de correção para avaliação do erro refrativo \\
sem a utilização da cicloplegia. Métodos: Foram estudados 623 pacientes \\
(1.246 olhos), de ambos os sexos, com idade entre 3 e 40 anos. As refra- \\
tometrias estática e dinâmica foram obtidas usando-se o refrator automá- \\
tico Shin-Nippon Accuref-K 9001. A cicloplegia foi obtida com a instila- \\
ção de uma gota de colírio ciclopentolato a 1\%, com refratometria estática \\
30 minutos após. Os dados foram submetidos à análise estatística usando \\
a técnica do modelo de regressão linear e modelo de regressão múltipla do \\
valor dióptrico com e sem cicloplegia, em função da idade. Resultados: \\
A correlação entre valores dióptricos sem e com cicloplegia quanto ao \\
erro astigmático variou de 81,52\% a 92,27\%. Quanto ao valor dióptrico \\
esférico, a correlação foi menor(53,57\% a 87,78\%). O mesmo se observou \\
em relação ao eixo do astigmatismo (28,86\% a 58,80\%). O modelo de \\
regressão múltipla em função da idade mostrou coeficiente de determi- \\
nação múltiplo maior para a miopia (86,38\%) e astigmatismo (79,79\%). O \\
menor coeficiente foi observado para o eixo do astigmatismo (17,70\%). \\
Conclusão: Avaliando-se os erros refrativos com e sem cicloplegia, \\
observou-se alta correlação nas ametropias cilíndricas. Foram desenvol- \\
vidas equações matemáticas como fator de correção para refratometrias \\
dos pacientes sem cicloplegia, portadores de ametropias cilíndricas e \\
esféricas.
\end{tabular}

Descritores: Erros de refração; Acomodação ocular; Miopia; Hipermetropia; Astigmatismo

\section{INTRODUÇÃO}

A acomodação resulta da mudança na forma do cristalino, por meio de alteração na sua curvatura e espessura central, modificando o poder dióptrico do olho ${ }^{(1)}$. A teoria clássica de Helmholtz, respeitada até hoje, propõe que o músculo ciliar, ao se contrair, produz relaxamento das fibras zonulares, aumentando a espessura e a curvatura do cristalino e, em consequência, aumentando o seu poder refrativo. Esta teoria foi questionada por Schachar e colaboradores ${ }^{(2)}$ o que não foi confirmado posteriormente ${ }^{(3-4)}$.

Durante a vida ocorre redução fisiológica da acomodação, sendo esta mudança atribuída à desidratação e esclerose do cristalino, mesmo que permaneça a integridade funcional do músculo ciliar ${ }^{(5)}$. O poder acomodativo varia com o envelhecimento e existe uma tolerância acomodativa em função das idades, que é a medida do esforço acomodativo assintomático para focar objetos próximos dos olhos. 
O exame no estado dinâmico, em pacientes com capacidade acomodativa, tende a subcorrigir defeitos hipermetrópicos e supercorrigir os miópicos, podendo produzir dados de aproveitamento errôneos, se estes pacientes não estiverem ciclopegiados.

A atropina, o ciclopentolato e a tropicamida atuam paralisando as fibras inervadas pelo parassimpático anulando, assim, a capacidade acomodativa.

A refratometria objetiva clássica é realizada através da retinoscopia. Esta importante técnica tem sido substituída em alguns consultórios oftalmológicos pelo refrator automático, cuja precisão é confiável e possibilita ao médico maior agilidade no atendimento, embora não dispense a cicloplegia ${ }^{(6-8)}$.

Seria interessante a existência de um fator de correção para ser aplicado em refratometrias dinâmicas, de modo a dispensar o uso de drogas cicloplégicas. Desta forma, poderia haver maior conforto para o paciente, rapidez nas consultas e adequada prescrição de lentes corretivas.

O objetivo do presente estudo foi obter este fator de correção.

\section{MÉTODOS}

Foram avaliados 623 pacientes (1.246 olhos), atendidos na Faculdade de Medicina de Botucatu, de ambos os sexos, com idade entre 3 e 40 anos.

As refratometrias estática e dinâmica foram obtidas usando-se sempre o mesmo refrator automático, modelo ShinNippon Accuref-K 9001.

Foi realizada a refratometria dinâmica, seguida pela instilação de uma gota de ciclopentolato a $1 \%$ com avaliação da refratometria estática 30 minutos após a administração do colírio.

O exame refrativo foi realizado pelo mesmo oftalmologista.

Foram excluídos os indivíduos portadores de fatores que pudessem influenciar na avaliação da refração como: defeitos corneanos, opacidades de meio, alterações retinianas, estrabismo, pacientes não colaborativos e parâmetros que estivessem fora da capacidade de atuação do equipamento.

Os dados foram analisados, usando o modelo de regressão linear para eixo e valor dióptrico cilíndrico, com e sem cicloplegia. Por meio de um modelo de regressão múltipla, analisou- se também o poder dióptrico com e sem cicloplegia, em função da idade.

\section{RESULTADOS}

Em relação à ametropia esférica, avaliando-se indivíduos por faixa etária, observou-se baixa correlação entre as refrações dinâmica e estática para os indivíduos com idade menor ou igual a 10 anos, e aumento gradual na faixa etária de 11 a 40 anos (Tabela 1).

O índice de correlação entre refratometrias dinâmica e estática para o astigmatismo apresentou-se alto $(>0,8)$ e praticamente constante em todas as faixas etárias (Tabela 2).

No eixo do astigmatismo, a correlação apresentou-se baixa entre as refratometrias dinâmica e estática, em todas as faixas etárias (Tabela 3 ).

A avaliação das ametropias com e sem cicloplegia, segundo o modelo de regressão múltipla em função da idade, mostrou coeficiente de determinação múltiplo maior para a miopia e astigmatismo. O menor coeficiente foi observado para o eixo do astigmatismo (Tabela 4).

\section{DISCUSS ÃO}

O refrator automático é um recurso propedêutico que tem sido muito utilizado para a avaliação dos erros refrativos. Entretanto, deve-se considerar a refratometria estática para que se tenha melhor acurácia ${ }^{(9)}$.

Este estudo foi realizado com o intuito de encontrar um fator de correção a ser aplicado para a realização de refratometria dinâmica, visando a compensação do mecanismo de acomodação e evitando a necessidade do uso de cicloplegia, uma vez que esta acarreta embaçamento visual, fotofobia e consequente prejuízo das atividades dos pacientes. A avaliação da refração dinâmica é influenciada pela idade, uma vez que a capacidade acomodativa diminui ao longo dos anos. Logo, os indivíduos foram separados em faixas etárias. Foram incluídos indivíduos abaixo dos 40 anos de idade, visto estes apresentarem poder acomodativo maior do que indivíduos acima desta faixa etária. Ademais, acima dos 40 anos, há alta

\begin{tabular}{|c|c|c|c|c|}
\hline Olho & Faixa etária & Modelo de regressão linear & Coeficiente determinação & Correlação \\
\hline \multirow[t]{4}{*}{ Direito } & $\leq 10$ anos & Esf Cicl $=0,4594$ Esf SCicl $+1,5242$ & 0,2870 & 0,5357 \\
\hline & 11 a 20 anos & Esf Cicl $=0,7629$ Esf SCicl $+1,1964$ & 0,6400 & 0,8002 \\
\hline & 21 a 30 anos & Esf $\mathrm{Cicl}=0,7648$ Esf SCicl $+0,8855$ & 0,5588 & 0,7475 \\
\hline & 31 a 40 anos & Esf Cicl $=0,8986$ Esf SCicl $+0,9675$ & 0,7706 & 0,8778 \\
\hline \multirow[t]{4}{*}{ Esquerdo } & $\leq 10$ anos & Esf $\mathrm{Cicl}=0,5123$ Esf SCicl $+1,4845$ & 0,3880 & 0,6229 \\
\hline & 11 a 20 anos & Esf Cicl $=0,6951$ Esf SCicl $+1,1990$ & 0,5819 & 0,7628 \\
\hline & 21 a 30 anos & Esf $\mathrm{Cicl}=0,8437$ Esf SCicl $+0,8038$ & 0,5699 & 0,7549 \\
\hline & 31 a 40 anos & Esf Cicl $=0,8453$ Esf SCicl $+0,9373$ & 0,6528 & 0,8080 \\
\hline
\end{tabular}




\begin{tabular}{|c|c|c|c|c|}
\hline Olho & Faixa etária & Modelo de regressão linear & Coeficiente determinação & Correlação \\
\hline \multirow[t]{4}{*}{ Direito } & $\leq 10$ anos & C Cicl $=0,8486$ C SCicl $+1,0048$ & 0,7168 & 0,8466 \\
\hline & 11 a 20 anos & C Cicl $=0,8346$ C SCicl $+1,4688$ & 0,6645 & 0,8152 \\
\hline & 21 a 30 anos & C Cicl $=0,8960$ C SCicl $+1,2708$ & 0,8544 & 0,9243 \\
\hline & 31 a 40 anos & C Cicl $=0,9444$ C SCicl $+0,0479$ & 0,8699 & 0,9327 \\
\hline \multirow[t]{4}{*}{ Esquerdo } & $\leq 10$ anos & C Cicl $=0,9424$ C SCicl - 0,0219 & 0,8102 & 0,9001 \\
\hline & 11 a 20 anos & C Cicl $=0,9053$ C SCicl $+0,0583$ & 0,8451 & 0,9193 \\
\hline & 21 a 30 anos & C Cicl $=1,0028$ C SCicl $+0,0388$ & 0,8380 & 0,9154 \\
\hline & 31 a 40 anos & C Cicl $=0,8381$ C SCicl $+0,0988$ & 0,7793 & 0,8828 \\
\hline
\end{tabular}

\begin{tabular}{|c|c|c|c|c|}
\hline Olho & Faixa etária & Modelo de regressão linear & Coeficiente determinação & Correlação \\
\hline \multirow[t]{4}{*}{ Direito } & $\leq 10$ anos & Ex Cicl = 0,3307 Ex SCicl + 42,4042 & 0,1041 & 0,3227 \\
\hline & 11 a 20 anos & Ex Cicl $=0,5761$ Ex SCicl $+23,3486$ & 0,3458 & 0,5880 \\
\hline & 21 a 30 anos & Ex Cicl $=0,4542$ Ex SCicl $+49,2039$ & 0,1864 & 0,4317 \\
\hline & 31 a 40 anos & Ex Cicl $=0,3868$ Ex SCicl $+38,3439$ & 0,1372 & 0,3704 \\
\hline \multirow[t]{4}{*}{ Esquerdo } & $\leq 10$ anos & Ex Cicl $=0,3426$ Ex SCicl $+48,7710$ & 0,1049 & 0,3239 \\
\hline & 11 a 20 anos & Ex Cicl $=0,3900 \mathrm{Ex} \mathrm{SCicl}+48,6523$ & 0,1393 & 0,3733 \\
\hline & 21 a 30 anos & Ex Cicl $=0,3033$ Ex SCicl $+51,6731$ & 0,0833 & 0,2886 \\
\hline & 31 a 40 anos & Ex Cicl $=0,5667 \mathrm{Ex} \mathrm{SCicl}+26,3907$ & 0,3184 & 0,5642 \\
\hline
\end{tabular}

\begin{tabular}{|c|c|c|}
\hline Grupo & Modelo de regressão & $\begin{array}{c}\text { Coeficiente } \\
\text { determinação múltiplo }\end{array}$ \\
\hline Miopia & Estática $=0,309323+0,898176 \times$ Dinâmica $-0,001189 \times$ idade $(p<0,001)(p>0,05)$ & $86,38 \%$ \\
\hline Hipermetropia & Estática $=1,835380+0,511020 \times$ Dinâmica $-0,020960 \times$ idade $(p<0,001)(p<0,0001)$ & $42,01 \%$ \\
\hline Eixo & Estática $=39,273700+0,433504 \times$ Dinâmica $+0,025562 \times$ idade $(p<0,0001)(p>0,05)$ & $17,70 \%$ \\
\hline Cilindro & Estática $=-0,053347+0,906162 \times$ Dinâmica $-0,001139 \times$ idade $(p<0,0001) \quad(p>0,05)$ & $79,79 \%$ \\
\hline \multicolumn{3}{|c|}{$\begin{array}{l}3 \text { ano } \leq \text { idade } \leq 40 \text { anos } \\
0 \leq \text { eixo sem cicloplegia } \leq 180 \\
-7,75 \leq \text { cilindro } \leq 0,00 \\
-11,62 \leq \text { miopia } \leq-0,25 \\
0,00 \leq \text { hipermetropia } \leq 9,00\end{array}$} \\
\hline
\end{tabular}

correlação entre as refrações dinâmica e estática ${ }^{(10)}$, justamente por causa da falência do sistema acomodativo. A correlação se mostra elevada em indivíduos afácicos e pseudofácicos, pois neste grupo de indivíduos a acomodação é nula ${ }^{(11)}$.

O tipo de erro refrativo também influencia nos resultados, sendo conhecido que há variação principalmente do erro refrativo esférico, com tendência à subcorreção dos defeitos hipermetrópicos e supercorreção dos miópicos. Esta afirmação se confirma quando se observa que a correlação entre os valores com e sem cicloplegia foi baixa nos erros esféricos, principalmente para os indivíduos mais jovens.

Em estudo prévio, foram examinadas 4.973 crianças chinesas, entre 7 e 18 anos, com uso refrator automático, antes e após a cicloplegia. Os resultados mostraram que o erro esférico apresentava-se mais negativo ou menos positivo antes da cicloplegia. As diferenças foram maiores para olhos hipermetrópicos (diferença significativa de $-2,98 \pm 1,65 \mathrm{D}$ ), menores para os olhos emétropes e menores ainda para olhos míopes (diferença significativa de $-0,41 \pm 0,46 \mathrm{D}$ ). Pequena diferença foi encontrada para medidas do astigmatismo com e sem cicloplegia $(0,08 \pm 0,13 \mathrm{D})^{(12)}$.

A correlação entre refratometrias estática e dinâmica com relação ao valor do astigmatismo foi alta. Em outro estudo, do qual fizeram parte indivíduos da mesma faixa etária, também não se observou variação importante do erro astigmático antes e após a cicloplegia ${ }^{(9)}$. 
Nos testes subjetivos com cicloplegia, há a desvantagem de a pupila permanecer dilatada, ocasionando fotofobia e aberrações ópticas que também interferem no exame refratométrico e, consequentemente, nos resultados. Este fator pode ter sido responsável pela baixa correlação observada neste estudo no eixo do astigmatismo, antes e após a dilatação.

Variações no eixo do astigmatismo foram observadas por outros quando foram avaliadas crianças sob retinoscopia antes e após a cicloplegia, principalmente em crianças com estrabismo $^{(13)}$.

Em outro estudo realizado com crianças chinesas entre 3 e 5 anos, foram comparadas as retinoscopias antes e após a ciploplegia. Observou-se que o erro refrativo com cicloplegia poderia ser aproximado multiplicando o erro esférico por 1,45 e adicionado $+0,39 \mathrm{D}$ ao produto, enquanto o poder do astigmatismo e o seu eixo permaneceram iguais ${ }^{(14)}$.

Nosso estudo visou o estabelecimento de um fator de correção para compensação do sistema acomodativo. Na tabela 4 estão apresentadas as equações que podem ser empregadas para este fim, contendo refração dinâmica e idade como variáveis.

Portanto, há formas de se ter o valor do erro refrativo sem que se empregue a cicloplegia. Entretanto, novos estudos devem ser providenciados a fim de tornar mais simples estes cálculos.

\section{CONCLUSÃO}

Avaliando-se os erros refrativos com e sem cicloplegia observou-se que há correlação alta nos erros astigmáticos. Foram desenvolvidas equações matemáticas como fator de correção para refratometrias dos pacientes sem cicloplegia, portadores de ametropias cilíndricas e esféricas.

\section{ABSTRACT}

Purpose: To determine a correction factor for refractive errors evaluated without cycloplegy effect. Methods: A study was made with 623 patients $(1,246$ eyes) of both sexes, aging between 3 and 40 years old. The dynamic and static refractometries were obtained using the automated refractor ShinNippon Accuref-K 9001. 1\% Cyclopentolate was dropped and the static refractometry was performed in 30 minutes. Data were analyzed using the linear regression model and the multi- ple regression model of the diopter values with and without cycloplegy effect according to age. Results: The correlation between the astigmatism diopter values with and without cicloplegy ranged from $81.52 \%$ to $92.27 \%$. Analyzing the spherical diopter values, the correlation was lower $(53.57 \%$ to $87.78 \%)$. The astigmatism axis also revealed low correlation values $(28.86 \%$ to $58.80 \%)$. The multiple regression model according to age demonstrated multiple determination coefficient with high values for myopia $(86.38 \%)$ and astigmatism (79.79\%). The lowest multiple determination coefficient was observed for astigmatism axis (17.70\%). Conclusion: It was possible to demonstrate a high correlation in refractive errors with and without cycloplegy effect on the cylindrical ametropies. Mathematical formules, for cylindrical and spherical ametropies, were presented as a correction factor for refraction of the patients not submitted to cycloplegy.

Keywords: Refraction errors; Ocular accommodation; Myopia; Hyperopia; Astigmatism

\section{REFERÊNCIAS}

1. Helmholtz HLF. Triatise on physiological optics. New York: Dover; 1962

2. Schachar RA, Black TD, Kash RL. The mechanism of accommodation and presbiopia in the primate. Ann Ophthalmol. 1995;27:58-67.

3. Glassner A, Kaufman PL. The mechanism of accommodation in primates. Ophthalmology. 1999;106(5):863-72.

4. Burd HJ, Judge SJ, Flavell MJ. Mechanism of accommodation of the human eye Vision Res. 1999;39(9):1591-5.

5. Alves, AA. Refração. Rio de Janeiro: Cultura Médica; 1989. p.67-8.

6. Bicas HEA, Alves AA, Uras R. Refratometria Ocular. São Paulo: Cultura Médica; 2005. p.350.

7. Alves, A.A. Refração. Rio de janeiro: Cultura Médica, 2000. p.470.

8. Salvesen S, Kohler M. Automated refraction. A comparative study of automated refraction with Nidek AR - 1000 autorefractor and retinoscopy. Acta Ophthalmol (Copenh). 1991;69(3):342-6.

9. Passos Jr WS, Manetti LD, Schellini SA, Padovani CR, Padovani CRP [Importance of cycloplegia in the objective auto-refraction and subjective refraction]. Rev Bras Oftalmol. 2005;64(6):371-5. Portuguese

10. Ghose S, Nayak BK, Singh JP. Critical evaluation of the NR-1000F auto refractometer. Br J Ophthalmol. 1986;70(3):221-6.

11. Isenberg SJ, Del Signore M, Madani-Becker G. Use of the HARK autorefractor in children. Am J Ophthalmol. 2001;131(4):438-41.

12. Zhao J, Mao J, Luo R, Li F, Pokharel GP, Ellwein LB. Accuracy of noncycloplegic autorefraction in school-age children in China. Optom Vis Sci. 2004; 81(1):49-55.

13. Bujara K, Schulz E, Haase W. [Retinoscopy under cycloplegic and non-cycloplegic conditions in children comparison of measurements of three examiners]. Albrecht Von Graefes Arch Klin Exp Ophthalmol. 1981;216(4):339-43. German

14. Chan OY, Edwards M. Comparison of cycloplegic and noncycloplegic retinoscopy in Chinese pre-school children. Optom Vis Sci. 1994;71(5):312-8. 